\title{
Botulinum Toxin in Children with Cerebral Palsy: An Update
}

\author{
Farid Hareb ${ }^{1}$ Carlo M. Bertoncelli ${ }^{2}$ Olivier Rosello ${ }^{3}$ Virginie Rampal ${ }^{3} \quad$ Federico Solla $^{3}$ \\ ${ }^{1}$ Clinique "Les Cadrans Solaires," 11 Route de Saint-Paul, 06140, \\ Vence, France \\ ${ }^{2}$ Fondation Lenval EEAP “H. Germain," 337 Les Provençales de \\ Ginestière, Nice, France \\ 3 Pediatric Orthopaedic Surgery, Fondation Lenval, 57 Avenue \\ Californie, Nice, France \\ Neuropediatrics 2020;51:1-5. \\ Address for correspondence Carlo M. Bertoncelli, PhD, Institut " $\mathrm{H}$. \\ Germain,", 337 Les Provençales de Ginestière, Nice, France \\ (e-mail: bertoncelli@unice.fr).
}

\begin{abstract}
Keywords

- botulinum toxin

- children

- cerebral palsy

- spasticity

Injected in a muscle, the botulinum toxin causes localized and temporary paralysis by acting on the neuromuscular synapse. Currently, it is widely prescribed for the treatment of limb spasticity in children from the age of 2 years. Combined with rehabilitation and other treatments, it helps to progress in motor learning, promotes functional progression, and delays orthopaedic degradations.

Numerous randomized, placebo-controlled studies have shown efficacy in reducing spasticity, improving passive and active mobility, reducing pain, and improving upper limb comfort care. The side effects are rare and commonplace.

The injection technique is accessible after specific training and practice. The indication is better evaluated by a multidisciplinary team. A precise clinical evaluation, assisted by an instrumental analysis (videography, spatiotemporal parameters, kinematics, kinetics, and electromyography), makes it possible to determine the aims of the treatment and to evaluate the outcome.

The objective of this review is to present current evidence base and practices regarding the use of botulinum toxin in children with cerebral palsy.
\end{abstract}

\section{Introduction}

Botulinum toxin is the treatment of spasticity with the highest level of scientific proof, used for $\sim 30$ years in cerebral palsy $(\mathrm{CP})$ patients.

Numerous randomized, placebo-controlled trials have shown efficacy in reducing spasticity, improving passive and active mobility, reducing pain, and improving comfort care.

Our objective is to present a review of the literature about botulinum toxin injection in patients with $\mathrm{CP}$ from infancy to late adolescence.

\section{Historical and Mode of Action}

Botulinum neurotoxins are produced by anaerobic Grampositive bacteria of the genus Clostridium, identified in the last century by Émile van Ermengem when describing botu- lism. Their blocking role in neuromuscular transmission was demonstrated in 1949 by Burgen et al. ${ }^{1}$ This ground-breaking discovery was the starting point of its indication in the treatment of focal dystonia first, and later of limb spasticity.

Normally, the nerve message is transmitted from the motor neurons to the muscles by the release of acetylcholine $(\mathrm{ACH})$ in the neuromuscular synapse. Injected in a muscle, the toxin causes localized and temporary paralysis by acting on the neuromuscular synapse. ${ }^{2}$ The absence of ACH in the synapse suppresses the affinity of the nicotinic receptors on the membrane of the muscle fibers, thus promoting the destruction of the receptors and the block of the neurotransmission. Prior to the resumption of synaptic functioning by synthesis of new receptors, a synaptic remodeling occurs. From the first days following the injection of botulinum
DOI https://doi.org/ 10.1055/s-0039-1694988. ISSN $0174-304 X$. (c) 2020 Georg Thieme Verlag KC Stuttgart · New York 
toxin, there is indeed a budding of new nerve endings from the terminal axon, which thus restores contact with the muscle, as it happens in reinnervated muscles after axon lesion. ${ }^{2}$ This process explains the reversible effect of an injection of botulinum toxin. Generally, muscle function returns to the baseline after 3 to 5 months.

\section{Clinical Use}

After providing the information on the expected effect and the possible side effects, the patient's and/or parents' approval is explicitly manifested.

Injection date, pain assessment through visual analogue scale, doses, and treated muscles are recorded.

The specialties Botox, Dysport, Neurobloc, Xeomin are available and indicated in neurological conditions.

Various premedication drugs are available, one at a time or in combination: intravenous ketamine, oral or intravenous benzodiazepine (e.g., midazolam), oral hydroxyzine, inhalation of an equimolar mixture of nitrous oxide and oxygen. ${ }^{3}$ Distraction techniques during the session help to relieve pain. ${ }^{4}$ An anesthetic ointment type EMLA should be placed at the injection site 60 ' before injection. ${ }^{5}$ In some difficult cases, a general anesthesia can be proposed.

The location of the muscles can be performed by palpation, by electromyogram (EMG) needle with injection route or ultrasound, especially in children, allowing a noninvasive identification of muscles and surrounding structures. ${ }^{5,6}$

\section{The Doses}

- The doses used depend on the weight of the patient, the number of muscles treated, the severity of the spasticity, the size of the muscle, and the type of toxin. The dose may be maximal in case of paralysis or severe deficit; conversely, it is minimal in case of active motor skills ${ }^{7}$

- The units are different and are not international unitsthere is no recognized equivalence: $1 \mathrm{~mL}$ for Botox, 100 Allergan units/mL, $2.5 \mathrm{~mL}$ for Dysport, 100 Speywood units per 1 to $2.5 \mathrm{~mL}$. The maximum recommended total dose for children is 20 Allergan units/kg for Botox and 300 units per session; for Dysport, 30 Speywood units/kg and 1000 units per session (professional agreement), in adults: 500 Allergan units for Botox and 1500 Speywood units for Dysport.

In the case of first injection and especially in patients with comorbidities, lower initial doses are recommended: for Botox, 3 to 8 units $/ \mathrm{kg}$ without exceeding 300 units per session; for Dysport, 10 units/ $\mathrm{kg}$ in case of unilateral injection and 20 units $/ \mathrm{kg}$ in case of bilateral injections without exceeding 1,000 units per session. ${ }^{7}$

An interval of 3 months minimum is required between two sessions.

\section{Data from the Literature}

A comprehensive review of the literature was conducted on PubMed with the following keywords: botulinum toxin, child and $\mathrm{CP}$ or spasticity. Six-hundred ninety articles were found.
One-hundred thirty-four abstracts were assessed. Sixty-six articles were selected for complete reading.

\section{Lower Limb}

Twenty-five studies were selected from levels 1 to 4 (8-31).

The age of children ranges from 6 months to 19 years; the toxins used are Botox, Dysport, or Xeomin.

Botulinum toxin injections in the triceps showed superior treatment effects in the reduction in spasticity and improvement in range of motion (ROM) and functional outcome measures in spastic diplegic children as compared with phenol blocks. $^{8}$

A significant decrease in spasticity is reported in all studies that specifically analyzed it. There was also a significant improvement in the ROM. ${ }^{8,9}$

One study found a decrease in pain. ${ }^{10}$

The functional level measured by the gross motor function measure has improved in 8 out of the 9 studies that were concerned, 5 of level $2,^{11-15}$ and 3 of level $3 .^{16-18}$

An improvement in the walking parameters or the ability to move has been found. ${ }^{9,13,16,19-21}$

The identification of the injection site is performed by simple anatomical palpation in six studies, and electrostimulation in two.

The dilution is most often 100 units/mL for Botox toxin, and 500 units/2.5 mL or 500 units/mL for Dysport toxin.

The duration of the effect is often evaluated at 3 to 4 months ( 4 weeks-24 months). ${ }^{20}$

One study shows that the younger the children, the greater the efficiency. ${ }^{13}$

One study shows a decrease in the use of tendon surgery. ${ }^{22}$

\section{Upper Limb}

Six studies were selected:6,23-27 3 level 2 and 3 level 4 , involving $\mathrm{CP}$ patients aged 2 to 19 years. The toxin used was always Botox.

Measurement of spasticity is reported in three studies: decreased in two, ${ }^{6,23}$ unchanged in one. ${ }^{24}$

A functional improvement has been measured with the following scales:

- Quality of upper extremity skills test ${ }^{25}$

- Pediatric Evaluation of Disability Inventory 25

- Goal attainment scaling. ${ }^{25-27}$

Two studies showed improvement in pain and nursing. An improvement in self-esteem has been reported once. The active function has not been studied.

Botox doses range from 1 to 12 units $/ \mathrm{kg}$. The dilution varies from 25 to 200 units/mL.

The majority of the authors used electrostimulation to locate the target.

The duration of the effect is of at least 3 months, and up to 12 months.

\section{Tolerance}

The different preparations share similar profile of safety. ${ }^{28}$

Side effects are rare and most often transient: pseudoinfluenza syndromes, rashes, cramps, pains, or hematomas at 
the site of injection. There is a risk of falling after triceps are injected, rare urinary leakage or retention after injection into adductors, disorders of swallowing after injection into neck muscles. $^{7}$

After repeated injections, the transient atrophy of the muscle is possible. However, there is no evidence of longterm myopathy or voluntary force decrease at follow-up. ${ }^{29}$

A functional aggravation can be linked to excessive contractions of the antagonist muscles and weakness of the agonist muscles or a technical error in locating the treated muscle.

Rarely, excessive muscle weakness and asthenia have been reported. Difficulty swallowing related to the spread of toxin away from the site of administration may lead to inhalation. The rare cases of reported fatalities involved children with multiple disabilities with unclear role of the toxin in the decease.

A correlation between dose and tolerance is shown in two studies; a preponderance of adverse effects for children under $45 \mathrm{~kg}$ was identified in only one study. ${ }^{30}$

One study focused on antibodies. High doses appear to be more likely to result in antibody formation. In another study, 117 patients with $\mathrm{CP}$ were followed for up to 2 years: the appearance of antibodies occurred in $28 \%$ of them; a quarter became resistant, $6 \%$ overall.

\section{Precautions}

The contraindications are as follows: myasthenia gravis, Lambert-Eaton syndrome, amyotrophic lateral sclerosis, use of anticoagulants, pregnancy and lactation, and major respiratory obstruction. ${ }^{7}$

In case of anesthesia and especially when using curarizing drugs, unusual effects are possible:

- Aggravation of the manifestations of botulism (aminoglycoside antibiotic treatment);

- Hypersensitivity to botulinum neurotoxin type A or any of the excipients.

The following interactions have been described:

- Aminoquinolines reduce the effect of botulinum toxin;

- Cyclosporine may potentiate the effect of botulinum toxin.

The best prevention remains the adherence to the recommendations of good practices: low doses in the first injection must be respected as well as the delays between the injections. ${ }^{7}$

\section{Indication in Children with Cerebral Palsy}

Spasticity reflects a velocity-dependent dynamic stiffness by an exaggeration of the stretching reflex, which can prevent movement, by its intensity and/or duration.

The multidisciplinary evaluation must answer two questions:

- Is spasticity embarrassing or helpful?

- Is annoying spasticity localized or diffuse? If it is localized, the indication of botulinum toxin injection is then easy; in case of diffuse spasticity, the injections are multisite before or in combination with other therapies.

\section{Indication in Preschool Children: Lower Limbs}

Treatment with toxin is often first-line.

The low weight of the child requires a limitation of the doses and the choice of the muscles to be treated. The dynamic equinus caused by the spasticity of the triceps sural is often the first indication in children, from the age of 2 years. The clinical examination confirms a tonic reflex at stretching on the gastrocnemius and/or the soleus; when walking, early activity of these muscles is confirmed. A videography can objectify the conditions of weight-bearing and the effect of the treatments. After the age of 3 years, EMG can be performed.

Spasticity of the long fibular can also be found in case of foot valgus; ${ }^{31}$ similarly, posterior tibial spasticity in frequent in case of foot varus. The toxin injection into these muscles may be associated with that of the triceps.

A supplement of treatment with antiequinus orthosis or casts is performed 2 to 3 weeks after the injections, time of the peak action of the botulinum toxins, if the dorsal ankle flexion has not normalized. A day and/or night orthoses is often proposed to maintain the gain. During the duration of action of the toxin, the physiotherapy sessions are continued or even increased in frequency

If there is spasticity of the hamstrings, rectus femoris, or adductors, the injections into these muscles are delayed until the second series of injections to reach sufficient doses. In case of hypertonicity on the adductors, a hip X-ray should be performed to search for a subluxation. The injections of toxins, associated with the physiotherapy, most often allow recovering a symmetrical abduction of the hips and a better tolerance of the orthoses. In case of spasticity of the hip flexors (more rare), the injection of iliopsoas is possible but requires a greater experience. The treatment targets the rectus femoris if there is stiff knee in extension with decreased oscillation and abnormal activity of this muscle at the EMG oscillation. Conversely, an inflected knee attitude is often associated with hamstring spasticity. The kinematic objective is the increase in the degree of flexion at the attack of the step and the EMG shows an early activity before the initial contact. The toxin injections are usually performed on semitendinosus and semimembranosus.

\section{Indication in Preschool Children: Upper Limbs}

From the age of 2 years, the grip can be hampered by a pronounced pronation, a thumb in the palm limiting the opening of the clamp, and a palmar flexion of the wrist. An ecological test, the Assisting Hand Assessment test, is available from the age of 18 months, and the Melbourne Unilateral Upper Limb Assessment from 5 years. The treatment targets the pronators, the intrinsic muscles of the thumb, and the flexors of the carp. The low doses of toxin needed to treat the troublesome hypertonia of these small muscles should prompt early investment in the upper limb. ${ }^{32,33}$

\section{Indication in School Age Children and Adolescents}

The multidisciplinary evaluation makes it possible to indicate toxin injection whenever the progress in reeducation and motor acquisitions is slowed down. On the other hand, 
the weight of the child allows the injection of several muscles at the same time, allowing a functional improvement. ${ }^{34,35}$ The injections are repeated once or twice a year for several years, depending on the benefits and tolerance, with further discussion of the doses and the choice of muscles at each session. It is often necessary to reduce an articular stiffness by successive immobilizations. ${ }^{36}$

If the result is insufficient, another therapy may be considered, for example, surgery. However, injections are often used as a test before functional orthopaedic surgery. In case of diffuse or severe hypertonia, especially in quadriplegia, oral treatment or neurosurgery can be proposed.

Contribution of the Quantified Analysis of the Movement After the age of 6 years, the quantified analysis shows the ambulation abnormalities, the repercussions of the spasticity on the movement, and the effectiveness of the treatments:

- Videography: reduction in sagittal disorders;

- Spatial and temporal parameters: increase in the walking speed, whether there is increase in the length and symmetry of the step or not;

- Improved kinematics and kinetics;

- EMG is often slightly modified.

Upper Limb

Treatment with toxin targets the following muscles:

- Superficial and/or deep flexor of fingers; a lower dose should be preferred in one of the muscles depending on the examination, to avoid weakening of the grip;

- Interosseous in case of intrinsic hand attitude with gooseneck;

- Round or square pronator in case of awkward pronation;

- Flexors of carp in case of stiffness in flexion;

- Elbow flexors: three muscles if the indication is comfort, one or two muscles if the indication is functional (often, brachial only);

- Shoulder muscles: lateral rotator muscles (infraspinatus, small round) or medial rotators (pectoralis major, subscapular).

\section{Trunk}

There is no evidence in favor of the protective role of the injection in the paravertebral muscles on the appearance of scoliosis, which is common in the CP patient. ${ }^{37,38}$

\section{Conclusion}

Botulinum toxin allows elective and reversible denervation by direct action on the motor plate. It is the treatment of choice for localized and troublesome spasticity. It is an integral part of the therapeutic arsenal for the $\mathrm{CP}$ motor disorders, always associated with rehabilitation and often with plasters, orthotics, and surgery. The side effects are mostly commonplace.

Currently, it is often prescribed in the treatment of spasticity from the age of 2 years. Associated with rehabilitation, it helps to make a course in motor learning; later, it promotes functional progression and delays orthopaedic degradations. Its use requires specific training and the support of a multidisciplinary team. A precise clinical evaluation, including the assessment of spasticity through validated and standardized scales, and an instrumental analysis (videography, spatiotemporal parameters, kinematics, kinetics and electromyography) make it possible to determine the objectives of the treatment and to evaluate the outcome.

\section{Financial support}

This research did not receive any specific grant from funding agencies in the public, commercial, or not-forprofit sectors.

\section{Conflicts of interest}

None. There are not potential conflicts of interest, real, or perceived; this includes a description of the role of the study sponsor(s). The authors report the following conflicts of interest:

FH, VR, CMB, FS: none.

$\mathrm{OR}$ received grants for attending congresses from Allergan.

Acknowledgments

Authors thank Miss Margherita Bellu from University of Cagliari, Italy, for proofreading.

\section{References}

1 Burgen AS, Dickens F, Zatman LJ. The action of botulinum toxin on the neuro-muscular junction. J Physiol 1949;109(1-2):10-24

2 Meunier FA, Schiavo G, Molgó J. Botulinum neurotoxins: from paralysis to recovery of functional neuromuscular transmission. J Physiol Paris 2002;96(1-2):105-113

3 Brochard S, Blajan V, Lempereur $M$, et al. Determining the technical and clinical factors associated with pain for children undergoing botulinum toxin injections under nitrous oxide and anesthetic cream. Eur J Paediatr Neurol 2011;15(04):310-315

4 Ben-Pazi H, Cohen A, Kroyzer N, et al. Clown-care reduces pain in children with cerebral palsy undergoing recurrent botulinum toxin injections - a quasi-randomized controlled crossover study. PLoS One 2017;12(04):e0175028. Doi: 10.1371/journal.pone.0175028. eCollection 2017.

5 Alter KE, Karp BI. Ultrasound guidance for botulinum neurotoxin chemodenervation procedures. Toxins (Basel) 2017;10(01):E18

6 Pitcher CA, Elliott CM, Panizzolo FA, Valentine JP, Stannage K, Reid SL. Ultrasound characterization of medial gastrocnemius tissue composition in children with spastic cerebral palsy. Muscle Nerve 2015;52(03):397-403

7 Traitement médicamenteux de la spasticité, recommandations de bonne pratique. French Agency for Drugs Security 2011 http:// ansm.sante.fr/var/ansm_site/storage/original/application/a79f07e ee915181bc9ae4e506140cecb.pdf Accessed 25 June 2018.

8 Gonnade N, Lokhande V, Ajij M, Gaur A, Shukla K. Phenol versus botulinum toxin $A$ injection in ambulatory cerebral palsy spastic diplegia: a comparative study. JPediatr Neurosci 2017;12(04): 338-343

9 Juneja M, Jain R, Gautam A, Khanna R, Narang K. Effect of multilevel lower-limb botulinum injections \& intensive physical therapy on children with cerebral palsy. Indian J Med Res 2017; 146(Supplement):S8-S14

10 Barwood S, Baillieu C, Boyd R, et al. Analgesic effects of botulinum toxin A: a randomized, placebo-controlled clinical trial. Dev Med Child Neurol 2000;42(02):116-121 
11 Baker R, Jasinski M, Maciag-Tymecka I, et al. Botulinum toxin treatment of spasticity in diplegic cerebral palsy: a randomized, double-blind, placebo-controlled, dose-ranging study. Dev Med Child Neurol 2002;44(10):666-675

12 Bjornson K, Hays R, Graubert C, et al. Botulinum toxin for spasticity in children with cerebral palsy: a comprehensive evaluation. Pediatrics 2007;120(01):49-58

13 Fazzi E, Maraucci I, Torrielli S, Motta F, Lanzi G. Factors predicting the efficacy of botulinum toxin-A treatment of the lower limb in children with cerebral palsy. J Child Neurol 2005;20(08):661-666

14 Hawamdeh ZM, Ibrahim AI, Al-Qudah AA. Long-term effect of botulinum toxin (A) in the management of calf spasticity in children with diplegic cerebral palsy. Eura Medicophys 2007;43 (03):311-318

15 Lee LR, Chuang YC, Yang BJ, Hsu MJ, Liu YH. Botulinum toxin for lower limb spasticity in children with cerebral palsy: a singleblinded trial comparing dilution techniques. Am J Phys Med Rehabil 2004;83(10):766-773

16 Bottos M, Benedetti MG, Salucci P, Gasparroni V, Giannini S. Botulinum toxin with and without casting in ambulant children with spastic diplegia: a clinical and functional assessment. Dev Med Child Neurol 2003;45(11):758-762

17 Hazneci B, Tan AK, Guncikan MN, Dincer K, Kalyon TA. Comparison of the efficacies of botulinum toxin $A$ and Johnstone pressure splints against hip adductor spasticity among patients with cerebral palsy: a randomized trial. Mil Med 2006;171(07): 653-656

18 Papavasiliou AS, Rapidi CA, Filiopoulos C, Rizou C, Skouteli HN. Evaluation of a multimodal management of prematurity-related spasticity. Pediatr Neurol 2006;35(06):400-407

19 Corry IS, Cosgrove AP, Duffy CM, McNeill S, Taylor TC, Graham HK. Botulinum toxin A compared with stretching casts in the treatment of spastic equinus: a randomised prospective trial. J Pediatr Orthop 1998;18(03):304-311

20 Koman LA, Brashear A, Rosenfeld S, et al. Botulinum toxin type a neuromuscular blockade in the treatment of equinus foot deformity in cerebral palsy: a multicenter, open-label clinical trial. Pediatrics 2001;108(05):1062-1071

21 Wang Y, Gao B. A dose-response relationship research on botulinum toxin type A local intramuscular injections of lower extremity spasticity in children with cerebral palsy. Childs Nerv Syst 2008;24(05):545-547

22 Metaxiotis D, Siebel A, Doederlein L. Repeated botulinum toxin A injections in the treatment of spastic equinus foot. Clin Orthop Relat Res 2002;(394):177-185

23 Pidcock FS. Botulinum toxin type A treatment in neurogenetic syndromes. Pediatr Rehabil 2005;8(04):298-302

24 Kawamura A, Campbell K, Lam-Damji S, Fehlings D. A randomized controlled trial comparing botulinum toxin A dosage in the upper extremity of children with spasticity. Dev Med Child Neurol 2007; 49(05):331-337
25 Lowe K, Novak I, Cusick A. Low-dose/high-concentration localized botulinum toxin A improves upper limb movement and function in children with hemiplegic cerebral palsy. Dev Med Child Neurol 2006;48(03):170-175

26 Russo RN, Crotty M, Miller MD, Murchland S, Flett P, Haan E. Upper-limb botulinum toxin A injection and occupational therapy in children with hemiplegic cerebral palsy identified from a population register: a single-blind, randomized, controlled trial. Pediatrics 2007;119(05):e1149-e1158

27 Wallen M, O'Flaherty SJ, Waugh MC. Functional outcomes of intramuscular botulinum toxin type a and occupational therapy in the upper limbs of children with cerebral palsy: a randomized controlled trial. Arch Phys Med Rehabil 2007;88(01):1-10

28 Carraro E, Trevisi E, Martinuzzi A. Safety profile of incobotulinum toxin $A[X e o m i n(\circledR)]$ in gastrocnemious muscles injections in children with cerebral palsy: randomized double-blind clinical trial. Eur J Paediatr Neurol 2016;20(04):532-537

29 Eek MN, Himmelmann K. No decrease in muscle strength after botulinum neurotoxin-a injection in children with cerebral palsy. Front Hum Neurosci 2016;10:506 eCollection 2016

30 Goldstein EM. Safety of high-dose botulinum toxin type A therapy for the treatment of pediatric spasticity. JChild Neurol 2006;21 (03):189-192

31 Boulay C, Jacquemier M, Castanier E, et al. Planovalgus foot deformity in cerebral palsy corrected by botulinum toxin injection in the peroneus longus: clinical and radiological evaluations in young children. Ann Phys Rehabil Med 2015;58(06):316-321

32 Sutcliffe TL, Gaetz WC, Logan WJ, Cheyne DO, Fehlings DL. Cortical reorganization after modified constraint-induced movement therapy in pediatric hemiplegic cerebral palsy. JChild Neurol 2007;22(11):1281-1287

33 Yang TF, Fu CP, Kao NT, Chan RC, Chen SJ. Effect of botulinum toxin type A on cerebral palsy with upper limb spasticity. Am J Phys Med Rehabil 2003;82(04):284-289

34 Boyd R, Graham HK. Botulinum toxin A in the management of children with cerebral palsy: indication and outcome. Eur J Neurol 1997;4(Suppl 2):S15-S22

35 Moleaners G, Desloovere K, Eyssen M, et al. Botulinum toxin type A treatment of cerebral palsy: an integrated approach. Eur J Neurol 1999;6(Suppl 4):S51-S57

36 Dai AI, Demiryürek AT. Serial casting as an adjunct to botulinum toxin type a treatment in children with cerebral palsy and spastic paraparesis with scissoring of the lower extremities. JChild Neurol 2017;32(07):671-675

37 Bertoncelli CM, Solla F, Loughenbury PR, Tsirikos AI, Bertoncelli D, Rampal V. Risk factors for developing scoliosis in cerebral palsy: a cross-sectional descriptive study. JChild Neurol 2017;32(07): 657-662

38 Bertoncelli CM, Bertoncelli D, Elbaum L, et al. Validation of a clinical prediction model for the development of neuromuscular scoliosis: a multinational study. Pediatr Neurol 2018;79:14-20 